\title{
Circular Polarisation in Star Forming Regions: The Origin of Homochirality?
}

\author{
P. W. Lucas, J. H. Hough \& A. C. Chrysostomou \\ Dept of Physical Sciences, University of Hertfordshire, College Lane, \\ Hatfield $A L 109 A B, U K$
}

\author{
J. A. Bailey \\ Anglo-Australian Observatory, Post Office Box 296, Epping, New South \\ Wales 121, Australia
}

\begin{abstract}
The origin of homochirality is one of the longest-standing puzzles in understanding the origins of life. In the laboratory, illumination by circularly polarised UV radiation (asymmetric photolysis) is an effective means of producing an enantiomeric excess in an otherwise racemic mix of chiral molecules. In the natural world, however, it has proven difficult to identify a suitable source of Circularly Polarised Light (CPL). Recent observations of L-excesses of $2-9 \%$ for a number of $\alpha$ methyl amino acids in the Murchison meteorite and our discovery of large degrees of CPL in some star forming regions has added weight to the suggestion that the origin of homochirality is extra-terrestrial. Here we report initial modelling of the production of that CPL.
\end{abstract}

\section{Physical Mechanisms for production of CPL}

An enantiomeric excess (ee) in chiral molecules can be produced by asymmetric photolysis (illumination by UV CPL), Bonner \& Dean 2000). In this work we examine the mechanisms which may produce the CPL observed in star formation regions at IR wavelengths (Bailey et al. 1998) and show initial results of models which calculate the degree of UV circular polarisation (CP) in the radiation field of a Young Stellar Object (YSO).

Starlight is generally unpolarised at the stellar surface. It can become polarised by interaction with dust grains in a nearby nebula. YSOs are surrounded by dusty nebulae, the remnant of their own birth cloud. Most stars tend to form in large clusters, which are pervaded by many additional dust clouds. Thus, chiral molecules near a young dwarf star (like the Sun 5 Gyr ago) may be illuminated by light from many other young stars and nearby nebulae.

High levels of CP are only produced if the dust grains are non-spherical and fairly well aligned with a common orientation. Each grain tends to align with the ambient magnetic field (B-field) such that the rotation axis with largest moment of inertia precesses around the direction of the B-field. Aligned grains are commonly observed in star forming regions via the linear polarisation (LP) by dichroic extinction. Aligned non-spherical grains can produce CP by 3 mechanisms, as described below. 
(1) Dichroic Scattering. A photon (particle of light) which is scattered by a non-spherical dust grain can gain high CP. This depends upon the direction of scattering and the orientation of the grain. Small deflection angles always produce low CP. Photons with wavelength significantly smaller than the size of the grain are nearly always deflected through small angles, producing low CP. Photons with wavelength much larger than the grain size will also have low CP after scattering, unless the grains are very absorptive, in which case there is little scattered light in any case (a low albedo). Hence, high CP is most likely to be produced if the wavelength is comparable to, or slightly larger than the typical grain size. The degree of CP produced by single scattering from spheroids has been modelled as a function of axis ratio and scattering angle by Gledhill \& McCall (2000).

Upom scattering, the (I,Q,U,V) Stokes vector of the photon defined relative to the scattering plane is modified by multiplying the $4 \times 4$ matrix Stokes matrix, $\mathbf{Z}$, whose 16 elements are functions of the scattering angles and grain orientation angles (3 independent angles for the oblate spheroids used in our calculation).

The I term in the Stokes vector is the intensity of the light, the $Q$ and $U$ terms represent LP along 2 axes inclined at 45 degrees each another. We are concerned with the $\mathrm{V}$ term, which describes the amount of CPL. The ratio V/I gives the degree of $\mathrm{CP}$, with positive $\mathrm{V}$ representing left-handed polarisation, and negative $\mathrm{V}$ representing right-handed polarisation, for a thumb pointing in the photon's direction of flight.

(2) Scattering followed by Dichroic Extinction. During flight throught a dusty medium, the polarisation state of a photon is modified by dichroic extinction. For unpolarised light this merely leads to LP parallel to the axis of grain alignment. However, if the light is already linearly polarised with a different position angle by prior scattering (even scattering by spherical or non-aligned grains) then dichroic extinction will produce CP. If the Stokes vector is defined relative to the axis of grain alignment (B-field axis), then the Stokes V term of the photon when travelling a distance $s$ through a uniform cloud with grain number density $n$ is modified by extinction as follows (Whitney \& Wolff 2002):

$V_{o b s}=\exp \left[-n K_{11} s\right] .\left\{V_{i n c} \cos \left(n K_{34} s\right)+U_{i n c} \sin \left(n K_{34} s\right)\right\}$

where $K_{11}$ and $K_{34}$ are elements of the $4 \times 4$ extinction matrix. Physically, Eq.(1) means that a phase difference is introduced between the components of the polarised part electromagnetic wave oscillating parallel to and perpendicular to the axis of grain alignment. The dusty nebula of a YSO can produce high $\mathrm{CP}$ by this mechanism. The mechanism operates most efficiently at wavelengths significantly larger than the grain size, where the ratio $K_{34} / K_{11}$ is high.

(3) Dichroic Extinction by grains aligned with a twisting B-field. This mechanism is very similar to the last. LP is converted to CP as the photon passes through the medium if the grain alignment axis is not parallel to the postion angle of the polarisation. Hence, dichroic extinction by aligned grains can generate LP from unpolarised light, and then if the grain alignment axis changes further along the flight path, CP will be produced as in Eq.(1). A twisting magnetic field would produce the necessary change in alignment axis. This mechanism can cause direct, unscattered light from a point source like 
a young star to gain CP. Again, the mechanism operates most efficiently at wavelengths significantly larger than the grain size.

\section{Monte Carlo modelling and Results}

We calculate the CP produced by a YSO nebula using the Monte Carlo method. Millions of photons are randomly generated at the stellar surface and each follows a random path through the nebula, perhaps scattering several times before it escapes the system or is absorbed. The details of the algorithm are described in Lucas (2002).

The millions of photons output from the system are used to construct polarimetric images of the nebula as seen in the far field (eg. from Earth). We also calculate the net CP received at points close to the nebula in the near field. The models shown here are for axisymmetric, 2-D, systems, since 3-D models are more computer intensive. Oblate spheroids are used since this is the easiest non-spherical shape to treat and generates higher CP than prolate spheroids with the same axis ratio. The grain axis ratio is $3: 1$ and perfect alignment is assumed. Such highly flattened grains appear to be necessary to reproduce the $17 \% \mathrm{CP}$ observed in Orion (Bailey et al. 1998) at IR wavelengths, which is attributed to dichroic scattering rather than extinction.

Results of some preliminary simulations are shown in Fig. 1. The model outputs are shown from a viewpoint slightly above the plane of the accretion disk $\left(\mathrm{i}=72^{\circ} .5\right)$ where the protostar itself is obscured. The calculations are for a wavelength of $0.22 \mu \mathrm{m}$. The envelope around the protostar has a radius of $5000 \mathrm{au}$ in this model. In all cases the flux is strongly concentrated towards the centre of the image, since the UV light tends to be scattered in a forward direction. These simulations use fairly clean (non-absorptive) grains with a range of sizes similar to interstellar dust. Larger grains tend to produce lower CP.

The results of these simulations show that high CP can be produced by a YSO in some scattering directions, due to a combination of dichroic scattering and dichroic extinction. However, the net CP at points in the near field is always below $5 \%$ in all simulations run to date. While there is still considerable parameter space to be explored, in terms of magnetic field structures, this suggests that an isolated YSO is unlikely to directly produce the enantiomeric excess (ee) of several percent, which has been reported for $\alpha$-methyl amino acids in meteorites (Cronin \& Pizzarello 1997). In the case of an axial field, which is probably realistic on scales of thousands of au (Tamura, Hough \& Hayashi 1995), the net CP is zero due to cancellation of the opposite senses of CP (RCP and LCP). We have artificially removed this cancellation by summing the CP received from just 2 or 3 quadrants of the image, as a simple way of modelling a highly asymmetric nebula. Even so, the net CP remains below $5 \%$ owing to the large amount of weakly polarised flux near the centre of the image. The less realistic helical field simulation has high $\mathrm{CP}$ in the lobes of the cavity, owing to dichroic extinction of scattered light. However, the lobes are too faint to greatly raise the net $C P$.

These results certaintly do not disprove a connection between CPL in star formation regions and the origin of homochirality. However, they suggest that 
the process is more indirect than simple asymmetric photolysis of prebiotic amino acids, as discussed by Bailey in this volume.
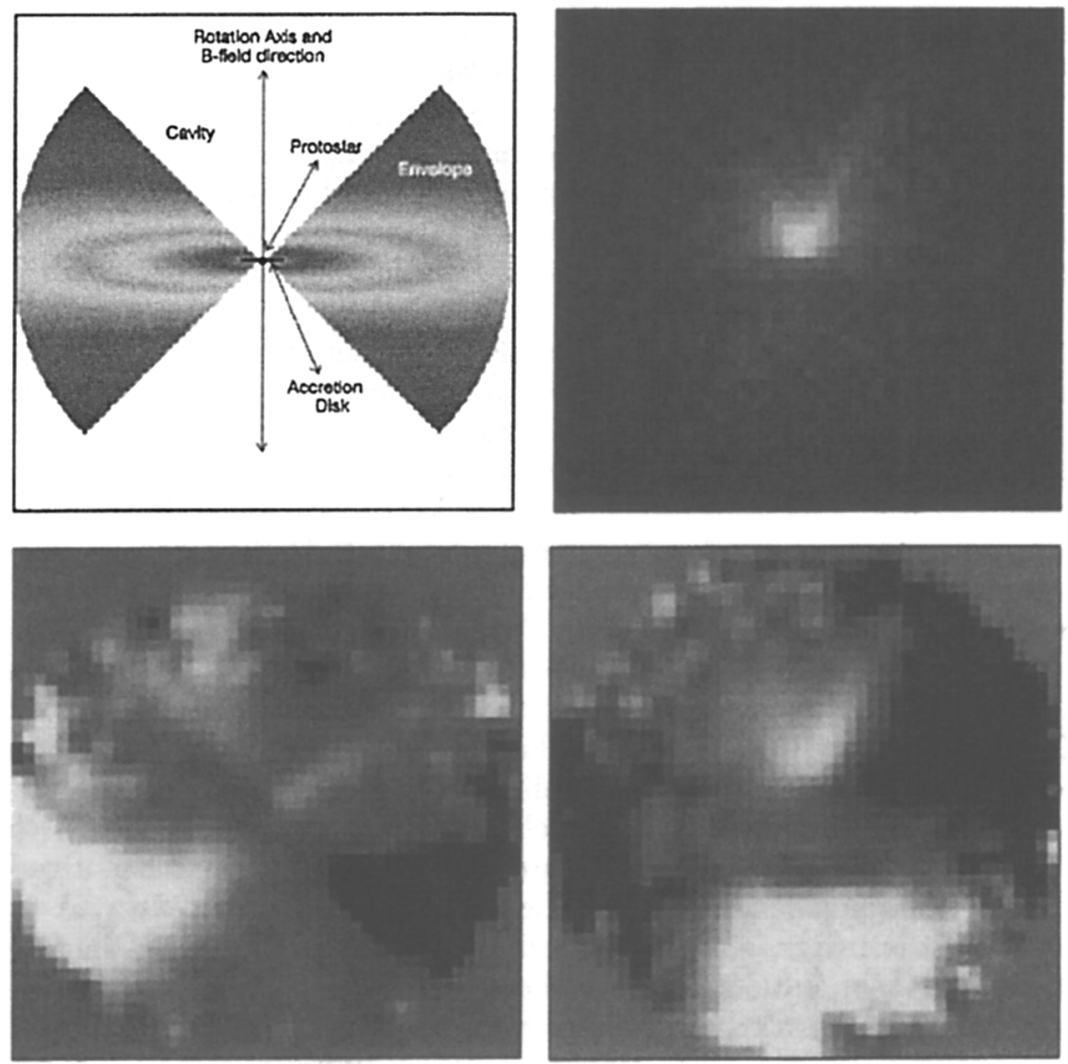

Figure 1. (top left) schematic of an axisymmetric YSO with an envelope, an evacuated bipolar cavity and an axial field. (top right) Model image for a helical field structure, with logarithmic stretch to show the faint nebulosity. (bottom left) Model CP map for an axial field, scaled between $-10 \%$ (right-handed)and $+10 \%$ (left-handed). (bottom right) Model CP map for a helical field with $45^{\circ}$ angle of ascension.

\section{References}

Bailey, J., et al. 1998, Science, 281, 672

Bonner, B. A., \& Dean, B. D. 2000, Orig. Life, 30, 513

Cronin, J. R., \& Pizzarello, S. 1997, Science, 275, 951

Gledhill, T. M., \& McCall, A. 2000, MNRAS, 314, 123

Lucas, P. W. 2002, J. Quant. Spec. \& Radiat. Transf., (in press), astro-ph/0208342

Tamura, M., Hough, J. H., \& Hayashi S. S. 1995, ApJ, 448, 346

Whitney, B. A., \& Wolff, M. J. 2002, ApJ, 574, 205 